\title{
A Note on Branch Flow Models with Line Shunts
}

\author{
Fengyu Zhou, Student Member, IEEE, and Steven H. Low, Fellow, IEEE
}

\begin{abstract}
When the shunt elements in the $\Pi$ circuit line model are assumed zero, it has been proved that branch flow models are equivalent to bus injection models and that the second-order cone relaxation of optimal power flow problems on a radial network is exact under certain conditions. In this note we propose a branch flow model that includes nonzero line shunts and prove that the equivalence and the exactness of relaxation continue to hold under essentially the same conditions as for zero shunt elements.
\end{abstract}

Index Terms-Branch flow model, DistFlow equations, line shunt, SOCP relaxation

\section{INTRODUCTION}

$\mathbf{T}$ HE DistFlow model is introduced in [1], [2] for radial networks. It is extended in [3], [4] to a branch flow model for general networks that may contain cycles. The branch flow model is justified in [5] by proving its equivalence to the widely used bus injection AC power flow model. For radial networks the DistFlow equations are nonlinear and hence optimal power flow (OPF) problems formulated based on these equations are nonconvex. Second-order cone programming (SOCP) relaxation is introduced in [3] and a sufficient condition for the relaxation to be exact is proved there for radial networks.

All models in [1], [2], [3], [5], [4] assume zero shunt elements (line charging) in the $\Pi$ circuit line model. Branch flow models are recently proposed in [6], [7] where the SOCP relaxation of a more conservative approximation of an OPF problem is studied. The purpose of this note is to propose an alternative branch flow model (equations (9) for radial networks and (11) for general networks) that includes nonzero line shunts, and prove that the equivalence and the exactness of SOCP relaxation continue to hold under essentially the same conditions as when line shunts are zero.

\section{BACKGROUND}

Notations. Let $\mathbb{C}$ denote the set of complex numbers and $\mathbb{R}$ the set of real numbers. Let $\mathbf{i}:=\sqrt{-1}$. For any $a \in \mathbb{C}$, $a^{*}$ denotes its complex conjugate. Unless otherwise specified a quantity $a$ denotes a vector whose $j$ th entry is $a_{j}$, e.g., $s:=\left(s_{j}, j \in N\right), S:=\left(S_{j k},(j, k) \in L\right)$. For vectors $a$ and $b$, $a \leq b$ means $a_{j} \leq b_{j}, \forall j$. If $a_{j}$ and $b_{j}$ are complex numbers,

This work was supported by NSF through grants CCF 1637598, ECCS 1619352, ECCS 1931662 and CPS ECCS 1739355.

Zhou and Low are with the Electrical Engineering Department, California Institute of Technology, Pasadena, CA 91125 USA (e-mails: \{f.zhou; slow\}@ caltech.edu) then the inequality is enforced for both real and imaginary parts.

Consider a single-phase power network with $N$ buses and $L$ lines modeled as a connected undirected graph $G(N, L)$ where $N:=\{1,2, \ldots, N\}$ and $L \subseteq N \times N$. This may represent the positive sequence network of a balanced three-phase system. A line $(j, k) \in L$, or $j \sim k$, is represented by a $\Pi$ circuit model $\left(y_{j k}^{s}, y_{j k}^{m}, y_{k j}^{m}\right)$ where $y_{j k}^{s}=y_{k j}^{s} \in \mathbb{C}$ are the series admittances of the line, $y_{j k}^{m} \in \mathbb{C}$ is the shunt admittance of the line at bus $j$, and $y_{k j}^{m}$ is the shunt admittance of the line at bus $k$. Since the line model may include not just transmission lines, but other devices such as transformers, we do not require $y_{j k}^{m}$ and $y_{k j}^{m}$ to be equal. Denote $z_{k j}=z_{j k}:=1 / y_{j k}^{s}$. Let

- $s_{j}:=p_{j}+\mathbf{i} q_{j}$ or $s_{j}:=\left(p_{j}, q_{j}\right)$ denote the real and reactive power injections at bus $j$.

- $V_{j}$ denote the voltage phasor at bus $j, j \in N$.

Without loss of generality [8], we fix bus 1 as the slack bus and set $V_{1}:=1 \angle 0^{\circ}$.

A widely used power flow model, we called a bus injection model (BIM), is specified by the following set of equations that relates nodal injections $s$ and voltages $V$ : for $j \in N$,

$$
s_{j}=\sum_{k: j \sim k}\left(y_{j k}^{s}\right)^{*}\left(\left|V_{j}\right|^{2}-V_{j} V_{k}^{*}\right)+\sum_{k: j \sim k}\left(y_{j k}^{m}\right)^{*}\left|V_{j}\right|^{2}
$$

Let $\mathbb{V}$ denote the set of power flow solutions:

$\mathbb{V}:=\left\{(s, V) \in \mathbb{C}^{2 N}:(s, V)\right.$ satisfies $\left.(1), V_{1}:=1 \angle 0^{\circ}\right\}$

While the bus injection model (1) involves only nodal variables, branch flow models also involve branch variables. Let

- $I_{j k}$ denote the phasor of the sending-end current from bus $j$ to bus $k,(j, k) \in L$.

- $S_{j k}:=P_{j k}+\mathbf{i} Q_{j k}$ or $S_{j k}:=\left(P_{j k}, Q_{j k}\right)$ denote the sendingend real and reactive power flows from bus $j$ to bus $k$, $(j, k) \in L$.

DistFlow equations. When the shunt elements are assumed zero $y_{j k}^{m}=y_{k j}^{m}=0$, [1], [2] introduce a power flow model, called DistFlow equations, for single-phase radial networks (i.e., networks with a tree topology) and uses it to optimize the placement and sizing of switched capacitors in distribution circuits for volt/var control. DistFlow equations adopt a directed graph. When the line is directed from bus $j$ to bus $k$, we refer to it by $j \rightarrow k \in L$. For each directed line $j \rightarrow k$, only the variable $S_{j k}$ is defined but not the variable $S_{k j}$. The orientation of the graph can be arbitrary, but for ease of exposition, we assume without loss of generality that bus 
1 is the root of the graph and every line points away from the root. The DistFlow equations are given by [1], [2]: ${ }^{1}$

$$
\begin{aligned}
s_{j} & =\sum_{k: j \rightarrow k} S_{j k}-\left(S_{i j}-z_{i j} \ell_{i j}\right), \quad j \in N \\
v_{j} \ell_{j k} & =\left|S_{j k}\right|^{2}, \quad j \rightarrow k \in L \\
v_{j}-v_{k} & =2 \operatorname{Re}\left(z_{j k}^{*} S_{j k}\right)-\left|z_{j k}\right|^{2} \ell_{j k}, \quad j \rightarrow k \in L
\end{aligned}
$$

Here the variable $v_{j}, \ell_{j k}$ are the squared voltage magnitude at bus $j$ and the squared current magnitude on line $j \rightarrow k$. The key feature of the DistFlow equations is that they do not involve angles of voltage and current phasors because these angles can be deduced from a solution $x:=(s, v, \ell, S) \in$ $\mathbb{R}^{3(N+L)}$ of the DistFlow equations (3) when the network is radial, but not when the network contains cycles.

Branch flow model. The model (3) is extended to a branch flow model (BFM) in [3], [4] for general networks that may contain cycles by introducing a cycle condition. Given any $x:=(s, v, \ell, S)$ define

$$
\beta_{j k}(x):=\angle\left(v_{j}-z_{j k}^{*} S_{j k}\right), \quad j \rightarrow k \in L
$$

and let $\beta(x):=\left(\beta_{j k}(x), j \rightarrow k \in L\right)$. Even though a solution $x$ of the DistFlow equations (3) does not contain voltage phase angles, $\beta_{j k}(x)$ can be interpreted as the angle difference across line $j \rightarrow k$ (also see Section III below). The DistFlow equations are extended in [3] to general networks as:

$$
\text { (3a)(3b)(3c), } \exists \theta \in \mathbb{R}^{N} \text { s.t. } \beta(x)=C^{\mathrm{T}} \theta
$$

where $C$ is the $N \times L$ incidence matrix of the directed graph with $C_{j l}=1$ if $l=j \rightarrow k$ for some $k,-1$ if $l=i \rightarrow j$ for some $i$, and 0 otherwise. We refer to the condition $\beta(x)=C^{\mathrm{T}} \theta$ on $x$ in (5) as the cycle condition, which can be enforced by introducing $\theta$ as additional variables. For general networks (3) can thus be interpreted as a relaxation of (5) where the cycle condition is ignored. When a network is radial the cycle condition is vacuous and (5) reduces to (3).

Equivalence and SOCP relaxation. A justification of the branch flow model (5), and hence (3), is that they are equivalent to the widely used bus injection model (1). Specifically it is proved in [5] that, when shunt elements are assumed zero $y_{j k}^{m}=y_{k j}^{m}=0$, there is a bijection between the set $\mathbb{V}$ and the set of solutions $x \in \mathbb{R}^{3(N+L)}$ of (5) for general networks and the set of solutions $x$ of (3) for radial networks.

The DistFlow equations (3a)(3c) are linear in its variable $x$ but (3b) is quadratic. Therefore OPF problems formulated using the DistFlow equations are nonconvex. Second-order cone program (SOCP) relaxation is introduced in [3] where (3b) is relaxed to the convex constraint:

$$
v_{j} \ell_{j k} \geq\left|S_{j k}\right|^{2}, \quad j \rightarrow k \in L
$$

The SOCP relaxation of an OPF problem is called exact if every solution of the relaxation attains equality in (6) and

\footnotetext{
1 The complex notation is used only as a shorthand for real quantities.
}

hence is optimal for the original OPF problem. It is proved in [3] that, for radial networks, SOCP relaxation is exact if the injections are not lower bounded.

Remark 1. A nodal shunt element at bus $j$ is included as an injection $s_{j}^{\text {sh }}$ in [1], [2], and as an admittance load $y_{j}^{\text {sh }}$ in [3]. They can be accommodated by modifying (3a) to:

$$
s_{j}+s_{j}^{\mathrm{sh}}=\sum_{k: j \rightarrow k} S_{j k}-\left(S_{i j}-z_{i j} \ell_{i j}\right)+y_{j}^{\mathrm{sh}} v_{j} .
$$

In this note we ignore nodal shunt elements for simplicity, but all results here will still hold when they are included.

The models in [1], [2] and [3], [4], however, assume zero shunt elements (line charging) $y_{j k}^{m}=y_{k j}^{m}=0$ in the $\Pi$ circuit model. In the rest of this note we generalize the branch flow models (3) and (5) to include line shunts, i.e., when $y_{j k}^{m}$ and $y_{k j}^{m}$ are nonzero, and show that the results in [3] [5] on equivalence and exact SOCP relaxation continue to hold under essentially the same conditions as when $y_{j k}^{m}=y_{k j}^{m}=0$.

\section{BRANCH FLOW MODEL WITH LINE SHUNTS}

Following [6] we consider the following branch flow model with line shunt elements in complex form (adopting an undirected graph):

$$
\begin{array}{rlrl}
s_{j} & =\sum_{k: j \sim k} S_{j k}, \quad j \in N & \\
S_{j k} & =V_{j} I_{j k}^{*}, \quad S_{k j}=V_{k} I_{k j}^{*}, & & (j, k) \in L \\
I_{j k} & =y_{j k}^{s}\left(V_{j}-V_{k}\right)+y_{j k}^{m} V_{j}, & & (j, k) \in L \\
I_{k j} & =y_{k j}^{s}\left(V_{k}-V_{j}\right)+y_{k j}^{m} V_{k}, & & (j, k) \in L
\end{array}
$$

where (7a) imposes power balance at each bus, (7b) defines branch power in terms of the associated voltage and current, and (7c)(7d) describes the Kirchhoff's laws. The main difference from the model in [3] is the use of undirected rather than directed graph when shunt elements are included so that line currents and power flows are defined in both directions.

Following [6], we define $\alpha_{j k}:=1+z_{j k} y_{j k}^{m}$ and $\alpha_{k j}:=1+$ $z_{k j} y_{k j}^{m}$ for line $(j, k) \in L$. Note that $\alpha_{j k}=\alpha_{k j}$ if and only if $y_{j k}^{m}=y_{k j}^{m}$ and $\alpha_{j k}=\alpha_{k j}=1$ if and only if $y_{j k}^{m}=y_{k j}^{m}=0$ as $\left|z_{j k}\right| \neq 0$. Substituting (7c)(7d) into (7b) gives

$$
\begin{aligned}
& V_{j} V_{k}^{*}=\alpha_{j k}^{*}\left|V_{j}\right|^{2}-z_{j k}^{*} S_{j k} . \\
& V_{k} V_{j}^{*}=\alpha_{k j}^{*}\left|V_{k}\right|^{2}-z_{k j}^{*} S_{k j} .
\end{aligned}
$$

This motivates the following generalization of (3) as a branch flow model for radial networks with shunt elements : for all $j \in N$ and $(j, k) \in L$,

$$
\begin{aligned}
s_{j} & =\sum_{k: j \sim k} S_{j k} \\
v_{j} \ell_{j k} & =\left|S_{j k}\right|^{2}, \quad v_{k} \ell_{k j}=\left|S_{k j}\right|^{2}, \\
\left|\alpha_{j k}\right|^{2} v_{j}-v_{k} & =2 \operatorname{Re}\left(\alpha_{j k} z_{j k}^{*} S_{j k}\right)-\left|z_{j k}\right|^{2} \ell_{j k} \\
\left|\alpha_{k j}\right|^{2} v_{k}-v_{j} & =2 \operatorname{Re}\left(\alpha_{k j} z_{k j}^{*} S_{k j}\right)-\left|z_{k j}\right|^{2} \ell_{k j} \\
\alpha_{j k}^{*} v_{j}-z_{j k}^{*} S_{j k} & =\left(\alpha_{k j}^{*} v_{k}-z_{k j}^{*} S_{k j}\right)^{*}
\end{aligned}
$$


The equivalence between (7) and (9) is provided as part of the proof to Theorem 1 in the Appendix.

Remark 2. We propose to use the original LinDistFlow model of [2] without shunt as a linear approximation of the branch flow model (9) as the effect of shunts is negligible; see justification in [9].

For general networks that may contain cycles, (8) suggests extending the definition (4) of $\beta(x)$ to both directions of a line as follows:

$$
\begin{array}{ll}
\beta_{j k}(x):=\angle\left(\alpha_{j k}^{*} v_{j}-z_{j k}^{*} S_{j k}\right), & (j, k) \in L \\
\beta_{k j}(x):=\angle\left(\alpha_{k j}^{*} v_{k}-z_{j k}^{*} S_{k j}\right), & (j, k) \in L
\end{array}
$$

To generalize (5), fix an arbitrary graph orientation represented by the incidence matrix $C$ and label the lines such that entries $\beta_{j k}(x)$ in the first half of the vector $\beta(x):=$ $\left(\beta_{j k}(x), \beta_{k j}(x), j \rightarrow k \in L\right)$ correspond to lines in the same direction as specified in $C$, and entries $\beta_{k j}(x)$ in the second half of $\beta(x)$ correspond to lines in the opposite direction. Then we propose the following generalization of (5) as a branch flow model for general networks with shunt elements: for all $j \in N$ and $(j, k) \in L$,

$$
\begin{aligned}
& (9 \mathrm{a})(9 \mathrm{~b})(9 \mathrm{c})(9 \mathrm{~d}) \\
& \exists \theta \in \mathbb{R}^{N} \text { s.t. } \beta(x)=[C,-C]^{\mathrm{T}} \theta
\end{aligned}
$$

where the components $\left(\beta_{j k}(x), \beta_{k j}(x)\right)$ of the vector $\beta(x)$ are ordered such that $(11 \mathrm{~b})$ implies

$$
\beta_{j k}(x)=\theta_{j}-\theta_{k}=-\beta_{k j}(x) .
$$

As $(9 b)(9 c)(9 d)$ imply that the magnitudes on both sides of (9e) are equal, (9e) can be replaced by (11b) for general networks. While $(9 \mathrm{e})$ is linear in the variable $x,(11 \mathrm{~b})$ is nonlinear. This is the major simplification of radial networks.

When the lines are modeled by series admittances without shunt elements, $y_{j k}^{m}=y_{k j}^{m}=0$, then $\alpha_{j k}=\alpha_{k j}=1$ and (9) and (11) can be shown to reduce to (3) and (5) respectively.

\section{EQUiVALENCE AND EXACT SOCP RELAXATION}

\section{A. Equivalence}

Even though BIM (1) and BFMs (9) and (11) are defined by different sets of equations in terms of their own variables, all of them are models of the Kirchhoff's laws and the Ohm's law and therefore must be related. We now clarify the precise sense in which these mathematical models are equivalent.

Let the sets of solutions of BFMs be:

$$
\begin{aligned}
\mathbb{X}_{\text {tree }} & :=\left\{x:=(s, v, \ell, S) \in \mathbb{R}^{3(N+2 L)} \mid x \text { satisfies }(9), v_{1}=1\right\} \\
\mathbb{X}_{\text {mesh }} & :=\left\{x:=(s, v, \ell, S) \in \mathbb{R}^{3(N+2 L)} \mid x \text { satisfies }(11), v_{1}=1\right\}
\end{aligned}
$$

Two sets $A$ and $B$ are said to be equivalent, denoted by $A \equiv B$, if there is a bijection between them. Indeed we explicitly exhibit bijections in the proof of Theorem 1 that preserve the physical meaning of variables in each set.
Theorem 1. $\mathbb{V} \equiv \mathbb{X}_{\text {mesh }} \subseteq \mathbb{X}_{\text {tree. }}$. For radial networks $\mathbb{V} \equiv$ $\mathbb{X}_{\text {mesh }}=\mathbb{X}_{\text {tree }}$.

Proof. See Appendix.

\section{B. Exact SOCP relaxation for radial networks}

Consider a radial network and the following OPF problem:

$$
\min _{x} f(x) \quad \text { s. t. } \quad x \in \mathbb{X}_{\text {tree }} \cap \mathbb{Y}
$$

where $\mathbb{Y}:=\{x: \underline{s} \leq s \leq \bar{s}, \underline{v} \leq v \leq \bar{v}, 0 \leq \ell \leq \bar{\ell}\}$ collects the operation constraints. We make the following assumptions:

A1 The cost function $f$ is strictly increasing in $\ell$, nondecreasing in $s$ and independent of $S$.

A2 The OPF problem (12) is feasible.

Common cost functions that satisfy A1 include real power loss or real power generation.

All the constraints in (12) are linear in $x$ except the quadratic equality (9b). Following [3] we relax (9b) to a second-order cone constraint: for $(j, k) \in L$,

$$
\left|S_{j k}\right|^{2} \leq v_{j} \ell_{j k}, \quad\left|S_{k j}\right|^{2} \leq v_{k} \ell_{k j}
$$

Let

$\mathbb{X}_{\mathrm{soc}}:=\left\{x \in \mathbb{R}^{3(N+2 L)} \mid x\right.$ satisfies $\left.(9 \mathrm{a})(13)(9 \mathrm{c})-(9 \mathrm{e}), v_{1}=1\right\}$

The SOCP relaxation of OPF (12) is:

$$
\min _{x} f(x) \quad \text { s. t. } \quad x \in \mathbb{X}_{\mathrm{soc}} \cap \mathbb{Y}
$$

Consider the following conditions and Theorem 2, which provides a sufficient condition for exact SOCP relaxation.

C1 $\underline{s}_{j}=-\infty-\mathbf{i} \infty$ for all buses $j \in N$.

C2 Both $\operatorname{Re}\left(\alpha_{j k}\right)$ and $\operatorname{Re}\left(\alpha_{k j}\right)$ are strictly positive for $j \sim k$.

Theorem 2. If $\mathrm{C} 1$ and $\mathrm{C} 2$ hold, then every optimal solution of the SOCP relaxation (14) is optimal for OPF (12).

Proof. If the optimal solution $\hat{x}=(\hat{s}, \hat{v}, \hat{\ell}, \hat{S})$ to (14) is not in $\mathbb{X}_{\text {tree }}$, then there must exist $j \sim k$ such that $\left|\hat{S}_{j k}\right|^{2}<\hat{v}_{j} \hat{\ell}_{j k}$. Taking the squared magnitude on both sides of (9e) and plugging in (9c),(9d), we obtain $\left|\hat{S}_{j k}\right|^{2}-\hat{v}_{j} \hat{\ell}_{j k}=\left|\hat{S}_{k j}\right|^{2}-\hat{v}_{k} \hat{\ell}_{k j}$ and therefore $\left|\hat{S}_{k j}\right|^{2}<\hat{v}_{k} \hat{\ell}_{k j}$ also holds. Following [3] we now construct another point $\tilde{x}=(\tilde{s}, \tilde{v}, \tilde{\ell}, \tilde{S})$ as follows.

$$
\begin{aligned}
\tilde{v} & =\hat{v}, & & \\
\tilde{\ell}_{j k} & =\hat{\ell}_{j k}-\operatorname{Re}\left(\alpha_{j k}\right) \varepsilon, & \tilde{\ell}_{k j} & =\hat{\ell}_{k j}-\operatorname{Re}\left(\alpha_{k j}\right) \varepsilon, \\
\tilde{S}_{j k} & =\hat{S}_{j k}-z_{j k} \varepsilon / 2, & \tilde{S}_{k j} & =\hat{S}_{k j}-z_{k j} \varepsilon / 2, \\
\tilde{s}_{j} & =\hat{s}_{j}-z_{j k} \varepsilon / 2, & \tilde{s}_{k} & =\hat{s}_{k}-z_{k j} \varepsilon / 2 .
\end{aligned}
$$

All the other entries of $\tilde{s}, \tilde{\ell}, \tilde{S}$ not listed above take the same values as in $\hat{s}, \hat{\ell}, \hat{S}$.

It can be easily checked that $\tilde{x}$ is feasible to (14) for sufficiently small $\varepsilon>0$. By A1, $f(\tilde{x})<f(\hat{x})$. It contradicts the optimality of $\hat{x}$.

Remark 3. The exactness of SOCP relaxation for DistFlow model without shunt elements is proved in [3], [4] under 
condition C1. Theorem 2 extends this result to DistFlow model with shunt elements under the additional condition C2. C2 generally holds since shunt admittances are usually much smaller than series admittances in magnitude and hence $\left|z_{j k} y_{j k}^{m}\right|$ and $\left|z_{k j} y_{k j}^{m}\right|$ are strictly smaller than 1 . The theorem hence suggests that exactness would hold even for applications where line shunts are not negligible.

\section{APPEndiX: Proof of TheOrem 1}

\section{Proof. Let}

$\tilde{\mathbb{X}}:=\left\{\tilde{x}:=(s, V, I, S) \in \mathbb{C}^{4(N+2 L)} \mid \tilde{x}\right.$ satisfies (7), $\left.V_{1}:=1 \angle 0^{\circ}\right\}$

For general networks that may contain cycles we will prove $\mathbb{V} \equiv \tilde{\mathbb{X}}, \tilde{\mathbb{X}} \equiv \mathbb{X}_{\text {mesh }}$, and $\mathbb{X}_{\text {mesh }} \subseteq \mathbb{X}_{\text {tree. }}$. For radial networks we will prove that $\mathbb{X}_{\text {mesh }}=\mathbb{X}_{\text {tree }}$.

Proof of $\mathbb{V} \equiv \tilde{\mathbb{X}}$. Given $(s, V) \in \mathbb{V}$, define $I$ by $(7 \mathrm{c})(7 \mathrm{~d})$ and $S$ by $(7 \mathrm{~b})$ and the resulting $(s, V, I, S) \in \tilde{\mathbb{X}}$. Conversely given $(s, V, I, S) \in \tilde{\mathbb{X}}$, substituting (7b)(7c)(7d) into (7a) shows $(s, V) \in \mathbb{V}$. As both mappings are derived from (7), it is easy to check that they are inverses of each other.

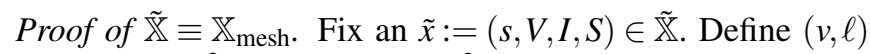
by $v_{j}:=\left|V_{j}\right|^{2}$ and $\ell_{j k}:=\left|I_{j k}\right|^{2}$. We now show that $x:=$ $(s, v, \ell, S) \in \mathbb{X}_{\text {mesh }}$. Clearly (9a)(9b) follow from (7a)(7b). For (9c) rewrite (7c) as $V_{k}=\alpha_{j k} V_{j}-z_{j k}\left(S_{j k} / V_{j}\right)^{*}$ where we have substituted $I_{j k}:=S_{j k}^{*} / V_{j}^{*}$ from (7b). Taking the squared magnitude on both sides gives

$$
v_{k}=\left|\alpha_{j k}\right|^{2} v_{j}+\left|z_{j k}\right|^{2} \ell_{j k}-2 \operatorname{Re}\left(\alpha_{j k}\left(z_{j k}\right)^{*} S_{j k}\right)
$$

which is $(9 \mathrm{c})$ (recall that $z_{j k}:=1 / y_{j k}^{s}$ ). Similarly for (9d). From (8) and the definitions of $\beta_{j k}(x)$ and $\beta_{k j}(x)$ in (10), we have

$$
\beta_{j k}(x)=\angle V_{j}-\angle V_{k}=-\beta_{k j}(x)
$$

and hence (11b) holds. This shows $x \in \mathbb{X}_{\text {mesh }}$. We denote this mapping from $\tilde{x}$ to $x$ as $\phi_{1}$.

Conversely fix an $x:=(s, v, \ell, S) \in \mathbb{X}_{\text {mesh }}$. Define $(V, I)$ as follows. Pick a $\theta$ that satisfies (11b) with $\theta_{1}=0 .{ }^{2}$ Let $V_{j}:=$ $\sqrt{v_{j}} e^{\theta_{j}}$ for $j \in N$. Define $I_{j k}$ and $I_{k j}$ in terms of $V$ according to (7c) and (7d) respectively for all lines $(j, k) \in L$. We now prove the following inequalities.

$$
\begin{aligned}
\left(\alpha_{j k}^{*} v_{j}-z_{j k}^{*} S_{j k}\right) & =\sqrt{v_{j} v_{k}} e^{\mathbf{i}\left(\theta_{j}-\theta_{k}\right)} \\
\left(\alpha_{k j}^{*} v_{k}-z_{k j}^{*} S_{k j}\right) & =\sqrt{v_{k} v_{j}} e^{\mathbf{i}\left(\theta_{k}-\theta_{j}\right)}
\end{aligned}
$$

Taking (15a) as an example, (10) and (11b) imply that both sides of (15a) have the same phase angle. We now show that both sides have the same magnitude as well. Indeed

$$
\begin{aligned}
\left|\alpha_{j k}^{*} v_{j}-z_{j k}^{*} S_{j k}\right|^{2} & =\left|\alpha_{j k}\right|^{2} v_{j}^{2}+\left|z_{j k}\right|^{2}\left|S_{j k}\right|^{2}-2 \operatorname{Re}\left(\alpha_{j k} z_{j k}^{*} v_{j} S_{j k}\right) \\
& =v_{j} v_{k}
\end{aligned}
$$

\footnotetext{
${ }^{2}$ Since $[C,-C]$ has rank $N-1$ and the all-ones vector is in its null space, such $\theta$ always uniquely exists.
}

where the last equality follows from multiplying both sides of (9c) by $v_{j}$ and then substituting $\left|S_{j k}\right|^{2}=v_{j} \ell_{j k}$ from (9b).

To show that $\tilde{x}:=(s, V, I, S) \in \tilde{\mathbb{X}}$ it suffices to show (7b) holds. By construction we have

$$
\begin{aligned}
V_{j} I_{j k}^{*} & =y_{j k}^{*}\left(v_{j}-V_{j} V_{k}^{*}\right)+\left(y_{j k}^{m}\right)^{*} v_{j} \\
& =y_{j k}^{*}\left(\alpha_{j k}^{*} v_{j}-\sqrt{v_{j} v_{k}} e^{\mathbf{i}\left(\theta_{j}-\theta_{k}\right)}\right) \\
& =y_{j k}^{*}\left(\left(\alpha_{j k}^{*} v_{j}-z_{j k}^{*} S_{j k}\right)-\sqrt{v_{j} v_{k}} e^{\mathbf{i}\left(\theta_{j}-\theta_{k}\right)}\right)+S_{j k} \\
& =S_{j k} .
\end{aligned}
$$

Here $y_{j k}$ is a shorthand for $y_{j k}^{s}$. Hence $\tilde{x}:=(s, V, I, S)$ satisfies (7) (the proof that $V_{k} I_{k j}^{*}=S_{k j}$ is similar). We denote the mapping from $x$ to $\tilde{x}$ as $\phi_{2}$. It is easy to check that $\phi_{1}$ and $\phi_{2}$ are inverses of each other and there is a bijection between $\tilde{\mathbb{X}}$ and $\mathbb{X}_{\text {mesh }}$.

Proof of $\mathbb{X}_{\text {mesh }} \subseteq \mathbb{X}_{\text {tree. }}$ Suppose $x \in \mathbb{X}_{\text {mesh }}$ and hence satisfies (11). Inequalities (15) imply (9e). Hence $x \in \mathbb{X}_{\text {tree }}$.

Proof of $\mathbb{X}_{\text {mesh }}=\mathbb{X}_{\text {tree }}$ for radial networks. Suppose $x \in$ $\mathbb{X}_{\text {tree }}$ and hence satisfies (9). We now show that $x$ satisfies (11b) when the network is radial. Recall that, by construction, the first half $\tilde{\beta}(x)$ of $\beta(x)$ correspond to lines in the same directions as specified by the incidence matrix $C$. The condition (9e) implies that $\beta(x)=\left[\tilde{\beta}^{\mathrm{T}}(x),-\tilde{\beta}^{\mathrm{T}}(x)\right]^{\mathrm{T}}$. It is well-known that $C$ has rank $N-1$ and $L=N-1$ for a (connected) radial network. Hence $C$ has full column rank and a solution $\theta$ to $\tilde{\beta}(x)=C^{\mathrm{T}} \theta$ always exists and is given by $\theta=C\left(C^{\mathrm{T}} C\right)^{-1} \tilde{\beta}(x)$. Therefore

$$
\beta(x)=\left[\tilde{\beta}(x)^{\mathrm{T}},-\tilde{\beta}(x)^{\mathrm{T}}\right]^{\mathrm{T}}=[C,-C]^{\mathrm{T}} \boldsymbol{\theta}
$$

which is condition (11b). Hence $x \in \mathbb{X}_{\text {mesh }}$.

This completes the proof of Theorem 1 .

\section{REFERENCES}

[1] M. E. Baran and F. F Wu. Optimal Capacitor Placement on radial distribution systems. IEEE Trans. on Power Delivery, 4(1):725-734, January 1989.

[2] M. E Baran and F. F Wu. Optimal Sizing of Capacitors Placed on A Radial Distribution System. IEEE Trans. on Power Delivery, 4(1):735743, January 1989.

[3] Masoud Farivar and Steven H. Low. Branch flow model: relaxations and convexification (parts I, II). IEEE Trans. on Power Systems, 28(3):2554 2572, August 2013.

[4] S. H. Low. Convex relaxation of optimal power flow, I: formulations and relaxations. IEEE Trans. on Control of Network Systems, 1(1):15-27, March 2014.

[5] Subhonmesh Bose, Steven H. Low, Thanchanok Teeraratkul, and Babak Hassibi. Equivalent relaxations of optimal power flow. IEEE Trans. on Automatic Control, 60(3):729-742, March 2015.

[6] Konstantina Christakou, Dan-Cristian Tomozei, Jean-Yves Le Boudec, and Mario Paolone. AC OPF in radial distribution networks - Parts I,II. arXiv:1503.06809v3, July 2016.

[7] Mostafa Nick, Rachid Cherkaoui, Jean-Yves Le Boudec, and Mario Paolone. An exact convex formulation of the optimal power flow in radial distribution networks including transverse components. IEEE Trans. on Automatic Control, 63(3):682-697, March 2018.

[8] A. J. Wood and B. F. Wollenberg. Power Generation, Operation, and Control. John Wiley \& Sons, Inc., 2nd edition, 1996.

[9] Fengyu Zhou and Steven H Low. A note on branch flow models with line shunts. arXiv:2003.10083, March 2020. 